\title{
RAPID COMMUNICATION The role of oxygen in increasing the carrier concentration of n-type silicon germanium-gallium phosphide alloys
}

\author{
D M Rowe, G Min and Y A Chen \\ School of Electrical, Electronics and Systems Engineering, University of Wales \\ College of Cardiff, Cardiff CF1 3YH, UK
}

Received 5 November 1993

\begin{abstract}
The role of oxygen in increasing the carrier concentraction of n-type silicon germanium-gallium phosphide alloys was investigated. It was found that silicon germanium-gallium phosphide alloy without additional dopant prepared by mechanical alloying and cold pressing is p-type if heat treated in argon or hydrogen, but is n-type if heat treated in air. The results indicate that carrier enhancement probably relates to the dissociation of gallium phosphide followed by oxidation of the dissociated gallium and phosphorus. A possible mechanism for carrier enhancement in n-type silicon germanium-gallium phosphide alloys is proposed.
\end{abstract}

In n-type silicon germanium alloys the thermoelectric figure-of-merit would optimize at a carrier concentration which is greater than the solubility of the dopant (phosphorus) in the alloys. Consequently, the maximum value of the figure-of-merit is not achieved using conventional doping techniques [1]. It has been reported that the thermoelectric properties on n-type silicon germanium alloys were improved by the addition of a few per cent of gallium phosphide and subjecting the material to high temperature heat treatment [2]. The improvement was accompanied by an increase in carrier concentration [3]. The current explanation of the improvement in the thermoelectric properties is that the addition of gallium phosphide increases the dopant solubility and enables the alloy to approach closer to its optiumum carrier concentration.

However, this explanation presents a problem. Gallium phosphide is a group III-V compound. The original purpose of introducing a small percentage of gallium phosphide was to reduce the thermal conductivity of the alloys [4]. In its compound state, gallium phosphide is a neutral impurity and does not contribute charge carriers. Furthermore, if gallium phosphide dissociates into gallium and phosphorus atoms following high temperature heat treatment, the phosphorus acts as a donor and gallium as an acceptor in silicon germanium alloys. Cross-doping is expected to occur, with the net carrier concentration depending on the difference between the number of phosphorus and gallium atoms. The experimental data of Cook et al [5] showed that the loss of gallium is always considerably less than of phosphorus. Consequently, in n-type silicon germanium alloys, the net carrier concentration would decrease, contrary to what is observed. The results of an experimental programme of work to provide an insight into the carrier enhancement mechanism is reported in this communication.

The specimens employed in this investigation were prepared by mechanical alloying and cold pressing. Elemental silicon (80\%) and germanium (20\%) with additional phosphorus, gallium, or gallium phosphide were ball milled using a Spex 8000 mill. The resulting powder was then cold pressed under a uniaxial pressure of $500-600 \mathrm{MPa}$, followed by isostatic pressing at about $300 \mathrm{MPa}$. Several undoped specimens, together with those doped with phosphorus, gallium, or gallium phosphide were prepared and designated SG, SGP, SGG and SGGP respectively. Half of the specimens containing each dopant type were heat treated at $1473 \mathrm{~K}$ in air, while the others were heat treated at $1473 \mathrm{~K}$ in an argon or hydrogen atmosphere. Both before and after heat treatment, the Seebeck coefficient and electrical resistivity were measured at room temperature using a hot probe and four probe apparatus respectively $[6,7]$. The conducting type of the specimens was given by the polarity of the Seebeck coefficient, while an estimate of the change in dopant concentration was obtained from the magnitude of the Seebeck coefficient and electrical resistivity.

The undoped specimens SG are intrinsic and heat treatment in different atmospheres has produced no 


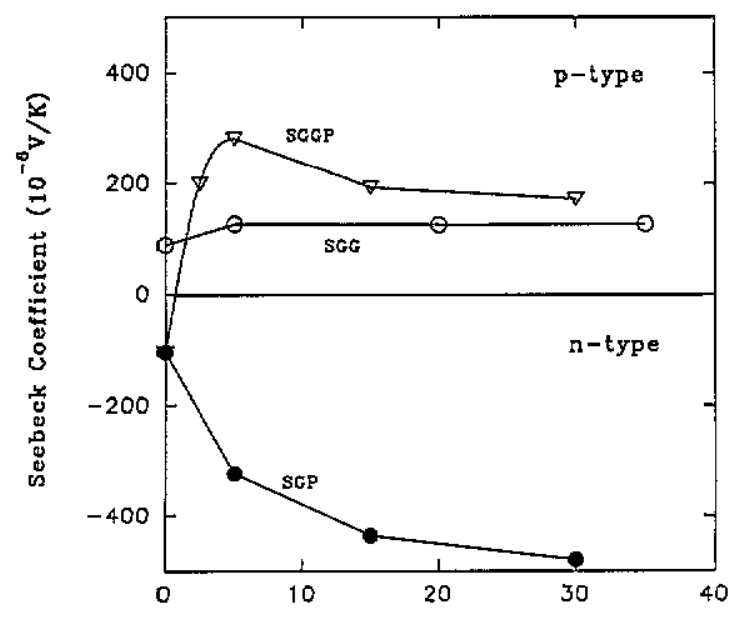

Heat treatment period (hrs)

Figure 1. Seebeck coefficients of specimens SGG, SGP and SGGP as a function of heat treatment period in argon atmosphere.

change in their intrinsic behaviour. However, aspressed specimens SGG are p-type, while specimens SGP and SGGP are n-type. In figure 1 is shown the changes in the Seebeck coefficient for specimens SGG, SGP and SGGP as a function of the period of heat treatment in argon. When heat treated $(1473 \mathrm{~K})$ in an argon atmosphere, sublimation of high vapour pressure materials is expected. It can be seen that the absolute values of the Seebeck coefficients for both SGG and SGP specimens were increased due to sublimation of the dopants. However, because the vapour pressure of phosphorus is greater than that of gallium, the Seebeck coefficients of specimens SGP increased more significantly than those of specimens SGG. Based on the variations of the Seebeck coefficients of specimens SGG and SGP, the changes in the Seebeck coefficients of specimens SGGP can be explained by dissociation of the gallium phosphorus, followed by a significant sublimation of the dissociated phosphorus. Since the loss of gallium is considerably less than that of phosphorus, more acceptors (gallium) will remain in the alloys and results in p-type material.

Sublimation and oxidation are expected to accompany the heat treatment of specimens in air. It can be seen from figure 2 that specimens SGG became intrinsic after heat treatment in air, while specimens SGP remained n-type. Gallium has a much lower vapour pressure than phosphorus. The intrinsic behaviour of specimens SGG can only be explained by the fact that gallium ions in silicon germanium have become electrically inactive, probably due to reaction with oxygen. On the other hand, little change in the Seebeck coefficients was observed in specimens SGP. This indicates a suppression of phosphorus sublimation, possibly also due to a reaction with oxygen. Consequently, the changes in the Seebeck coefficients of specimens SGGP after heat treatment in air can be explained in terms of the dissociation of gallium phosphide, followed by recombination of dissociated gallium and phosphorus with oxygen.

A possible bonding arrangement of the silicon germanium-gallium phosphide matrix is shown in

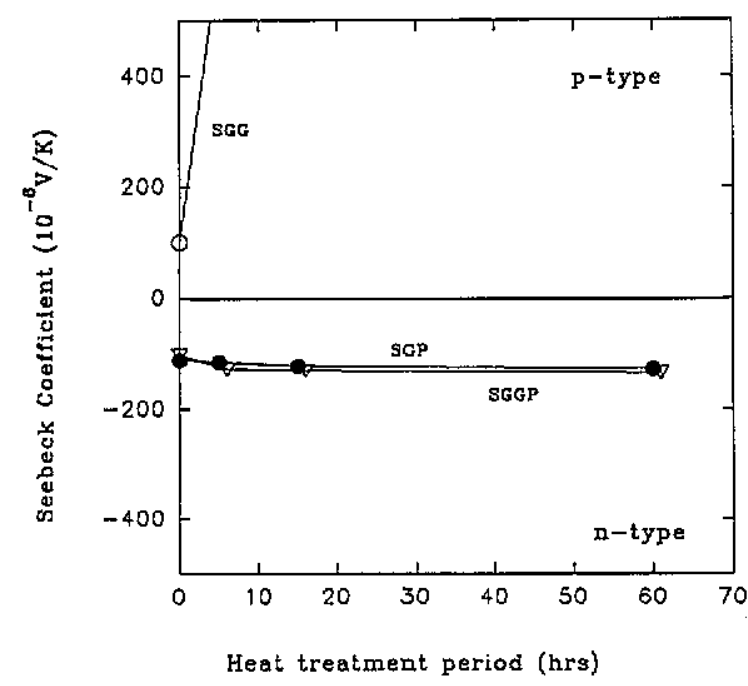

Figure 2. Seebeck coefficients of specimens SGG, SGP and SGGP as a function of heat treatment period in sir.

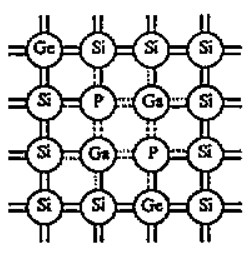

(a)

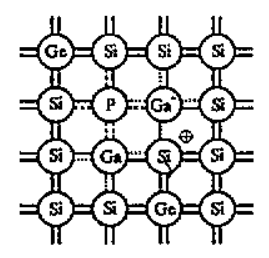

(b)

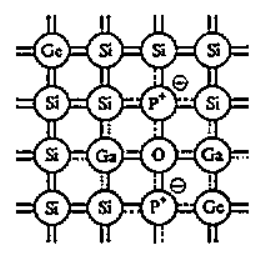

(c)

$$
\text { — Silicon and germanium bondings ........ Gallium bonding }
$$

Figure 3. Bonding arrangement in SiGe-GaP alloys:

(a) before heat treatment, (b) after heat treatment in argon, (c) after heat treatment in air.

figure 3. When gallium and phosphorus are tightly bonded into gallium phosphide, which is distributed in the silicon germanium matrix, one empty valence bond of the gallium atom is filled by the fifth electron of phosphorus. Neither gallium nor phosphorus contributes charge carriers to the valence or conduction bands. Tightly bonded gallium phosphide is a neutral impurity and alloys doped with gallium phosphide will be intrinsic as shown in figure 3(a).

During high temperature heat treatment the gallium phosphide dissociates, and the dissociated gallium and phosphorus provide acceptors and donors. If there are an equal number of dissociated gallium and phosphorus atoms, the dopant behaviour remains intrinsic due to compensation of the donors and acceptors. However, because the vapour pressure of gallium is always considerably less than that of phosphorus, more gallium (acceptors) would remain in the alloys after heat treatment in an argon atmosphere. In this situation, the electrical conductivity of the alloy is controlled by acceptors and the alloy is p-type as shown in figure 3(b).

If heat treatment is carried out in air, the alloys are exposed to oxygen. Since an oxygen atom possesses 6 valence electrons, one oxygen atom is likely to react 
with two gallium atoms to satisfy the empty valence bonds. This results in a reduction in the number of electrically active acceptors. However, the dissociated phosphorus will remain as donors and therefore the alloy is n-type. Furthermore, if a bond is formed between oxygen and phosphorus, the sublimation of phosphorus will be considerably less significant because the phosphorus-oxygen bond $(\mathrm{P}-\mathrm{O})$ is much stronger than the phosphorus-silicon (P-Si) or phosphorusgermanium $(\mathrm{P}-\mathrm{Ge})$ bonds. This is illustrated in figure 3(c).

Gallium phosphide has a cubic zinc sulphide crystal structure, which is compatible with the silicon germanium matrix. The solubility of gallium phosphide in silicon germanium alloys has been reported to be as high as $8 \%$ [4], which is much larger than that of phosphorus. The results of the experiments reported above suggest that the dissociated gallium and phosphorus may react with oxygen forming gallium-oxygen-phosphorus (Ga-O-P) clusters after high temperature heat treatment in air as shown in figure 3(c). The resulting Ga-O-P clusters form a structure which is also compatible with the silicon germanium matrix. However, the phosphorus donors in the $\mathrm{Ga}-\mathrm{O}-\mathrm{P}$ clusters differ from the phosphorus donors introduced by conventional doping. Consequently, the number of $\mathrm{Ga}-\mathrm{O}-\mathrm{P}$ clusters in the alloys is unlikely to be limited by the solubility of 'normal' phosphorus dopants. Therefore, in the case of silicon germanium-gallium phosphide alloys with additional phsophorus doping, the carrier concentration consists of two contributions: $\mathrm{Ga}-\mathrm{O}-\mathrm{P}$ donors and donors associated with the conventional phosphorus dopant. Consequently, the carrier concentration can be increased in n-type silicon germanium-gallium phosphide alloys to beyond the 'normal' solubility limits of phosphorus.
It is apparent that oxygen has played an important role in increasing the carrier concentration of n-type silicon germanium-gallium phosphide alloys. Carrier enhancement cannot be achieved in the alloys which are prepared completely free of oxygen. On the other hand, if the alloys are exposed to too much oxygen, dioxides of silicon or germanium will form around grain boundaries and the carrier mobility will be inevitably reduced. Evidently, the amount of oxygen in the alloys is important. Future work is planned in which silicon germanium-gallium phosphide material with controlled amounts of oxygen will be prepared.

\section{Acknowledgments}

This work is supported by the United Kingdom Science and Engineering Research Council. YAC acknowledges the financial support of the Brazilian Scientific Council. The authors would also like to thank Dr J K Luo for many helpful discussion.

\section{References}

[1] Rosi F D 1968 Solid-State Electron. 11 833-68

[2] Vandersande J W, Wood C and Draper S 1987 Proc. Mater. Res. Soc. Symp. vol 97 (New York: American Institute of Physics) pp 347-52

[3] Wood C 1987 Proc. 1st European Conf. on Thermoelectrics ed D M Rowe (London: Peter Peregrinus) pp 1-8

[4] Pisharody R K and Garvey L P 1978 Proc, 13th Int. Energy Conv. Eng. Conf. (New York: IEEE) pp 1963-8

[5] Cook B A, Harringa J L, Beaudry B J and Han S H 1992 11 th Int. Conf. on Thermoelectrics (Arlington, TX, USA) pp 28-32

[6] Cowles L E and Dauncey L A 1962 J. Sci. Instrum. 39 16-8

[7] Valdes L B 1955 Proc. IRE 42 420-5 